\title{
Confine sulfur in double-hollow carbon sphere integrated with carbon nanotubes for advanced lithium-sulfur batteries
}

\author{
Maru Dessie Walle ${ }^{1,2}$ (D) You-Nian Liu ${ }^{2}$
}

Received: 2 September 2020 / Accepted: 6 December 2020 / Published online: 2 January 2021

(c) The Author(s) 2021

\begin{abstract}
The lithium-sulfur $(\mathrm{Li}-\mathrm{S})$ batteries are promising because of the high energy density, low cost, and natural abundance of sulfur material. Li-S batteries have suffered from severe capacity fading and poor cyclability, resulting in low sulfur utilization. Herein, S-DHCS/CNTs are synthesized by integration of a double-hollow carbon sphere (DHCS) with carbon nanotubes (CNTs), and the addition of sulfur in DHCS by melt impregnations. The proposed S-DHCS/CNTs can effectively confine sulfur and physically suppress the diffusion of polysulfides within the double-hollow structures. CNTs act as a conductive agent. S-DHCS/CNTs maintain the volume variations and accommodate high sulfur content $73 \mathrm{wt} \%$. The designed S-DHCS/ CNTs electrode with high sulfur loading $\left(3.3 \mathrm{mg} \mathrm{cm}^{-2}\right)$ and high areal capacity $\left(5.6 \mathrm{mAh} \mathrm{mg} \mathrm{cm}{ }^{-2}\right)$ shows a high initial specific capacity of $1709 \mathrm{mAh} \mathrm{g}^{-1}$ and maintains a reversible capacity of $730 \mathrm{mAh} \mathrm{g}^{-1}$ after 48 cycles at $0.2 \mathrm{C}$ with high coulombic efficiency (100\%). This work offers a fascinating strategy to design carbon-based material for high-performance lithium-sulfur batteries.
\end{abstract}

Keywords Energy storage $\cdot$ Lithium-sulfur battery $\cdot$ Polysulfide $\cdot$ Double-hollow carbon sphere

\section{Introduction}

Researchers have been investigating to meet the increasing demand for high capacity energy storage devices, as well as looking for new technologies that will help to minimize the effects of global warmings, such as reducing energy consumption or facilitating the transition to renewables [1, 2]. In recent years, $\mathrm{Li}-\mathrm{S}$ battery has been attracted much attention because of its high energy density $\left(2600 \mathrm{Wh} \mathrm{kg}^{-1}\right)$, lower environmental impacts, low manufacturing costs, abundant supply of materials, ease of processing, and reduced environmental footprint [3-5]. However, Li-S battery has been firstly hampered by the low electrical conductivity of both sulfur $\left(5 \times 10^{-30} \mathrm{~S} \mathrm{~cm}^{-1}\right)$ and the discharge product $\left(\mathrm{Li}_{2} \mathrm{~S}\right)$, which lead to restricting the electron transport and the utilization of the active sulfur [6]. Second, the dissolution of

Maru Dessie Walle

marshet2014@yahoo.com

1 Department of Industrial Chemistry, College of Science, Bahir Dar University, Bahir Dar 79, Ethiopia

2 College of Chemistry and Chemical Engineering, Central South University, Changsha 410083, Hunan, People's Republic of China polysulfides in the conventional organic electrolyte $\left(\mathrm{Li}_{2} \mathrm{~S}_{x}\right.$, $4 \leq x \leq 8$ ) provides low coulombic efficiency and short cycle life $[7,8]$. Third, the volume change of sulfur (about 80\%) during the charge-discharge process leads to the pulverization of sulfur cathode and gives up a rapid capacity decay $[9,10]$.

Carbon materials have been intensively used as hosts for sulfur because they can increase the electrical conductivity of sulfur and immobilize the soluble polysulfides [11, 12]. However, due to the presence of weak interactions between sulfur and the carbon materials, the carbon-based cathode suffers from a capacity decay over long-term cycling. Besides, owing to their tendency to adsorb soluble polysulfides, polar metal oxide $\left(\mathrm{MnO}_{2}\right)$ [13], metal $\mathrm{WS}_{2}-\mathrm{WO}_{3}$ heterostructure [14], and metal sulfide $\left(\mathrm{MoS}_{2}\right)$ [15] also have been developed as sulfur hosts. However, the polar inorganic metallic compounds possess poor electrical conductivity, leading to limit the electron transport and diminish the electrochemical performance of the batteries [16]. In these aspects, it is still very important to design carbon-based electrodes which can provide adequate space for the storage of the active materials and soluble polysulfides, leading to fast electron transport and oxidation-reduction reaction.

People Repubic of China

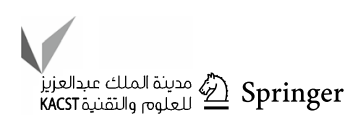


It has been confirmed that combining porous carbon with sulfur increase the cyclic performance and rate capability of Li-S battery. Besides, sulfur with graphene [17], carbon nanotubes [18], hollow carbon nanofibers [19], and conducting polymers [20] are the most common ways to enhance the electronic conductivity of sulfur and also as volume change barriers. The hollow structures have received much attention as hosting of large amount of sulfur. This leads to accommodate the volume expansion of the sulfur. Specially, the double-hollow structure enhances the advantages of hollow material, which could confine, store sulfur and retard the outward diffusion of soluble polysulfides. Hence, integrating the DHCS with carbon nanotubes will improve the conductivity and also helps to transfer the electron and ions. For example, Lou et al. found that the double-shelled hollow carbon spheres can effectively confine sulfur and also improved the accommodation of volume variation [21].

In this work, we report a rational design and synthesis of confining sulfur in double-hollow carbon spheres integrated with carbon nanotubes (S-DHCS/CNTs). The CNTs serve as a conducting agent and support the DHCS. The combination of DHCS and CNTs accelerates the electron transport and enhances the kinetics of the electrochemical reactions. The synergistic effects provide an outstanding cycling performance of S-DHCS/CNTs with high sulfur content (73\%) and high loading $\left(3.3 \mathrm{mg} \mathrm{cm}^{-2}\right)$.

\section{Experimental}

\section{Preparation of DHCS/CNTs composites}

The preparation of DHCS/CNTs is concisely illustrated in Fig. 1. In a typical experiment, $0.3 \mathrm{~g}$ resorcinol was dispersed into a solution containing $3 \mathrm{~mL}$ of tetraethyl orthosilicate (TEOS), $60 \mathrm{~mL}$ methanol, $20 \mathrm{~mL}$ distilled water, $0.2 \mathrm{~g}$ carbon nanotubes (CNTs), and $0.1 \mathrm{~g}$ sodium dodecyl sulfate (SDS). Afterward, $3 \mathrm{~mL}$ of ammonia solution (30 wt\%) was added, and stirred for $30 \mathrm{~min}$. Subsequently, a $1 \mathrm{~mL}$ formaldehyde solution was added to the dispersion under stirring. After $5 \mathrm{~h}$, the above steps were repeated without the addition of CNTs. $\mathrm{SiO}_{2} @ \mathrm{RF} @ \mathrm{SiO}_{2} @ \mathrm{RF} / \mathrm{CNT}$ composites were collected by centrifugation and washed three times with distilled water and methanol, and dried at $110{ }^{\circ} \mathrm{C}$ for $8 \mathrm{~h}$. The DHCS/CNTs composites were obtained by pyrolysis $\mathrm{SiO}_{2} @$ $\mathrm{RF} @ \mathrm{SiO}_{2} @ \mathrm{RF} / \mathrm{CNTs}$ at $800{ }^{\circ} \mathrm{C}$ for $2 \mathrm{~h}$ under argon atmosphere with a heating rate of $5{ }^{\circ} \mathrm{C} \mathrm{min}^{-1}$. DHCS/CNTs were obtained by etching the as-product with $2 \mathrm{M} \mathrm{NaOH}$ aqueous solution for $5 \mathrm{~h}$.

\section{Preparation of S-DHCS/CNTs and S-CNTs composites}

The S-DHCS/CNTs and S-CNTs composites were prepared with melt impregnations under pressure. A mixture of sulfur and DHCS/CNTs powder ( $8: 1$ by weight) was placed in a Teflon-lined steel autoclave and heated to $155^{\circ} \mathrm{C}$ for $8 \mathrm{~h}$. The preparation process of S-CNTs was the same as the method mentioned above.

\section{Materials characterization}

The morphology of the as-prepared materials was characterized by transmission electron microscopy (TEM, FEI Titan G2 60-300, USA). Thermogravimetric analysis (TGA, SDT Q600 V8.0, TA Instruments, USA) was conducted under an argon atmosphere to determine the sulfur content in the composites. The crystalline of sulfur was studied with X-ray diffraction (XRD, Rigaku, Dmax/2550VB, Japan) with $\mathrm{Cu}$ $\mathrm{K} \alpha$ radiation. The surface composition and chemical bonds were investigated by X-ray photoelectron spectroscopy (ThermoFisher Scientific, ESCALAB 250XI, USA).

\section{Electrochemical measurements}

The electrochemical measurements of the cathodes made from S-DHCS/CNTs and S-CNTs were carried out using CR2025 coin-cells with lithium foil as an anode, fabricated in an argon-filled glove box. The active materials (S-DHCS/ CNTs and S-CNTs) were mixed with acetylene black (AB) and polytetrafluoroethylene (PTFE) binder uniformly in a mass ratio of 8:1:1 in isopropyl alcohol, respectively. The prepared cathode membrane was cut and compressed at $10 \mathrm{MPa}$ onto stainless-steel wire mesh with $0.8 \mathrm{~cm}$ in diameter. The sulfur loading of the cathode is from 3.0 to

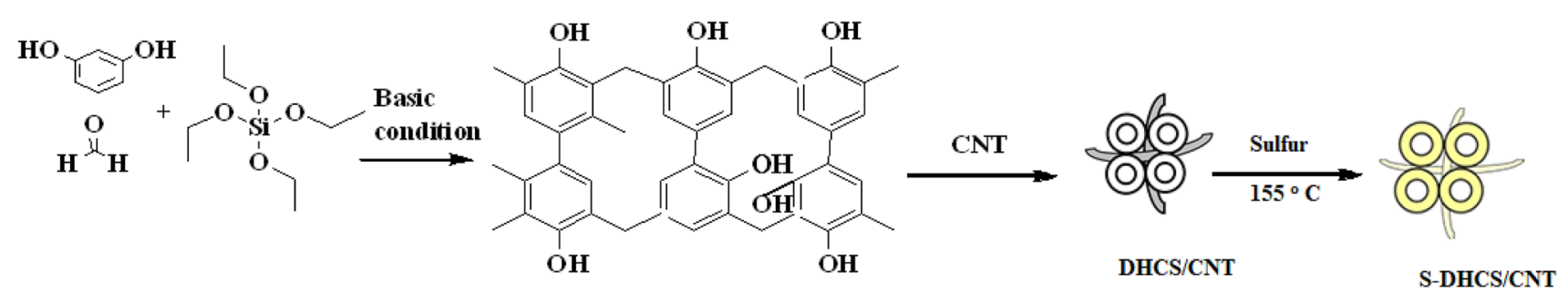

Fig. 1 Schematic illustrations for the preparation of S-DHCS/CNTs composite 
$3.3 \mathrm{mg} \mathrm{cm}^{-2}$. Celgard 2300 used as the separator and lithium bis(trifluoromethane) sulfonamide $(1.0 \mathrm{M})$ (LiTFSI, Novolyte Technology Co., Suzhou, China) in 1,3-dioxolane (DOL) and 1,2-dimethoxyethane (DME) (2:1 by weight) containing $1 \mathrm{wt} \%$ of $\mathrm{LiNO}_{3}$ as the electrolyte. The amount of electrolyte added was $50 \mathrm{~mL} \mathrm{~g}^{-1}$ for every cathode. Cyclic voltammetric measurement was performed with an electrochemical workstation (CHI 660E, Chenhua, Shanghai, China) in a voltage range from 1.5 to $3.0 \mathrm{~V}$ at a scan rate of $0.1 \mathrm{mV} \mathrm{s}^{-1}$. Galvanostatic charge-discharge tests were conducted with a LAND CT2001A battery test system (Jinnuo Electronic Co., Wuhan, China). All specific capacities of the cathode were calculated according to the weight of sulfur in the active material.

\section{Results and discussion}

The double-hollow carbon spheres with carbon nanotubes composites (DHCS/CNTs) have been prepared by polymerization of resorcinol with formaldehyde under the basic medium. The TEOS was added to form a hard template
$\left(\mathrm{SiO}_{2}\right)$ by in situ method. RF resin is used as a carbon precursor. Moreover, the CNTs are added to serve as a conducting and supporting agent. After carbonization, $\mathrm{SiO}_{2}$ was removed by etching in aqueous sodium hydroxide. Sulfur is added to DHCS/CNTs with melt impregnations (see in Fig. 1).

The morphology of the S-DHCS/CNTs composites was studied with transmission electron microscopy (TEM) (see Fig. 2). The unique hollow spheres with two shells interconnected with CNTs have been observed and the inner space of DHCS/CNTs is dark after sulfur loading as shown in Fig. 2a. The high-resolution TEM image of S-DHCS/CNTs also shows a porous structure (see Fig. 2b). The S-DHCS/ CNTs spheres reveal the diameter from 50 to $200 \mathrm{~nm}$. The two concentric shells supported with CNTs furnishing an excellent carbon matrix for high sulfur loading. To determine the sulfur content in S-DHCS/CNTs and S-CNTs composites, thermogravimetric analysis (TGA) was executed. The sulfur content in the S-CNTs and S-DHCS/CNTs is $62 \%$ and $73 \%$, respectively, as indicated in Fig. 2c. The higher sulfur content in S-DHCS/CNTs manifests the formation of strong sulfur binding interaction with the host material
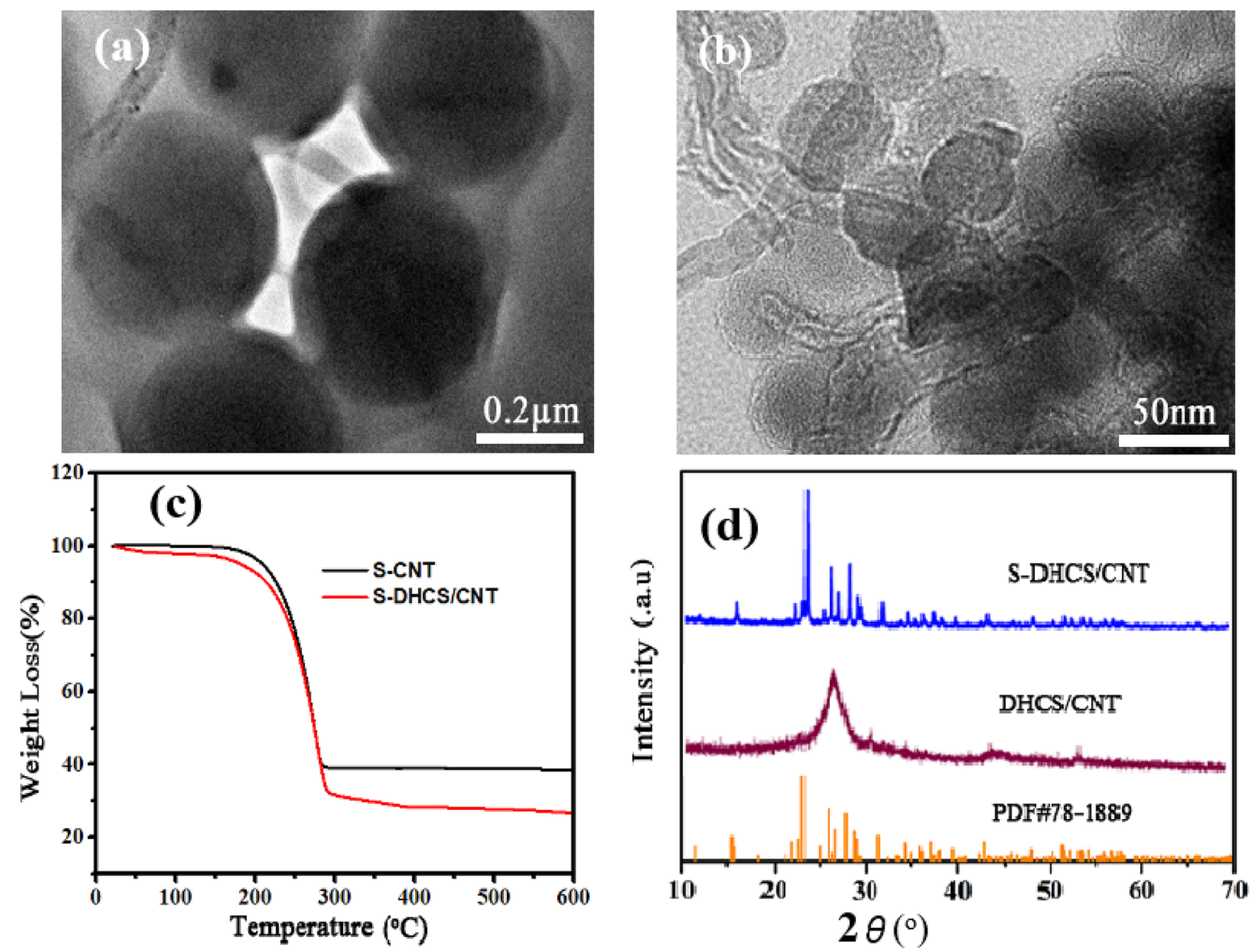

Fig. 2 TEM image of S-DHCS/CNTs at a low, b high magnifications. $\mathbf{c}$ TGA of S-DHCS/CNTs and S-CNTs. $\mathbf{d}$ XRD of DHCS/CNTs and S-DHCS/CNTs 
with high evaporation temperature [22]. This confirms that DHCS/CNTs have strong physical interactions with sulfur. The crystal structure of S-DHCS/CNTs was also investigated with X-ray diffraction (Fig. 2d). The characteristic peaks at $26^{\circ}$ and $43^{\circ}$ are attributed to the graphitic carbon [23], resulted due to the carbonization of the material at high temperatures. The characteristic peaks of the sublimed sulfur (PDF \# 78-1889) can be observed in the S-DHCS/CNTs, confirming the presence of sulfur in the material.

$\mathrm{X}$-ray photoelectron spectroscopy (XPS) was adopted to further verify the surface composition of S-DHCS/CNTs (see Fig. 3). In the survey XPS spectrum of S-DHCS/ CNTs (Fig. 3a), two obvious peaks centering at 284.8 and $532.5 \mathrm{eV}$, correspond to $\mathrm{C} 1 s$ and $\mathrm{O} 1 s$ [24], respectively. Besides, the peaks at 169 and $232 \mathrm{eV}$ are represented the $\mathrm{S} 2 p$ and $\mathrm{S} 2 s$, respectively. The obtained atomic percentages of carbon, oxygen and sulfur in S-DHCS/CNTs are $77.45 \%, 19.49 \%$ and $3.05 \%$, respectively. C $1 s$ peak deconvoluted into three species, corresponding to the $\mathrm{C}-\mathrm{C} / \mathrm{C}=\mathrm{C}$ bonds with $s p^{3}$ and $s p^{2}$ hybridizations $(284.5 \mathrm{eV})$, the covalent $\mathrm{C}-\mathrm{S}$ bond formed upon sulfurization $(285.3 \mathrm{eV})$, $\mathrm{C}-\mathrm{O}(286.4 \mathrm{eV})$ and the $\mathrm{O}-\mathrm{C}=\mathrm{O}(288.2 \mathrm{eV})$ [25]. The high-resolution $\mathrm{O} 1 s$ spectrum of S-DHCS/CNTs shows the interactions of oxygen with carbon, sulfur and hydrogen (see Fig. 3c). As illustrated in Fig. 3d, the high-resolution $\mathrm{S} 2 p$ peak which consists of two doublets at $165.1 \mathrm{eV}$ $\left(\mathrm{S} 2 p_{1 / 2}\right)$ and $163.9 \mathrm{eV}\left(\mathrm{S} 2 p_{3 / 2}\right)$ suggests the presence of $\mathrm{S}-\mathrm{S}$ species [26]. The $\mathrm{S}-\mathrm{O}$ peak at $168.6 \mathrm{eV}$ could result from a certain amount of sulfate existing in the sample because of the oxidation of sulfur in the air.

The cyclic voltammetry profile of the cell is illustrated in Fig. $4 \mathrm{a}$ at the scan rate of $0.1 \mathrm{mV} \mathrm{s}^{-1}$, showing the oxidation-reduction processes. Two prominent cathodic peaks at 2.30 and $2.02 \mathrm{~V}$ could be attributed to the reduction of sulfur to higher-order soluble lithium polysulfides $\left(\mathrm{Li}_{2} \mathrm{~S}_{n}, 4 \leq n \leq 8\right)$ and ultimately to insoluble $\mathrm{Li}_{2} \mathrm{~S}_{/} \mathrm{Li}_{2} \mathrm{~S}_{2}$, respectively. In the succeeding anodic scan, only one peak at about $2.46 \mathrm{~V}$ is observed, as a result of the oxidation of $\mathrm{Li}_{2} \mathrm{~S}$ to lithium polysulfides [16, 27, 28]. During the first four cycles, there are no significant changes for both anodic and cathodic peaks, confirming the high reversibility and electrochemical stability of the cathode. This validated that the unique structure S-DHCS/CNTs effectively confines the soluble polysulfides and maintains high utilization of the active material in the multistep reactions.
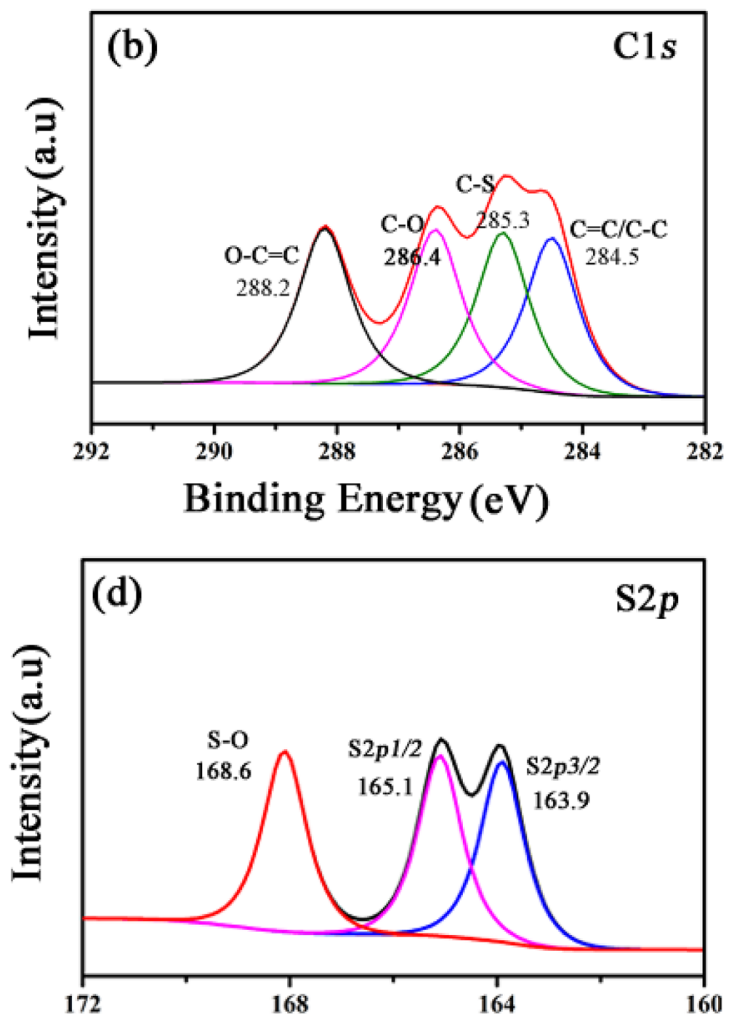

Binding Energy (eV)

Fig. 3 XPS spectra of a survey, b C $1 s$, c O $1 s$, and d S $2 p$ 

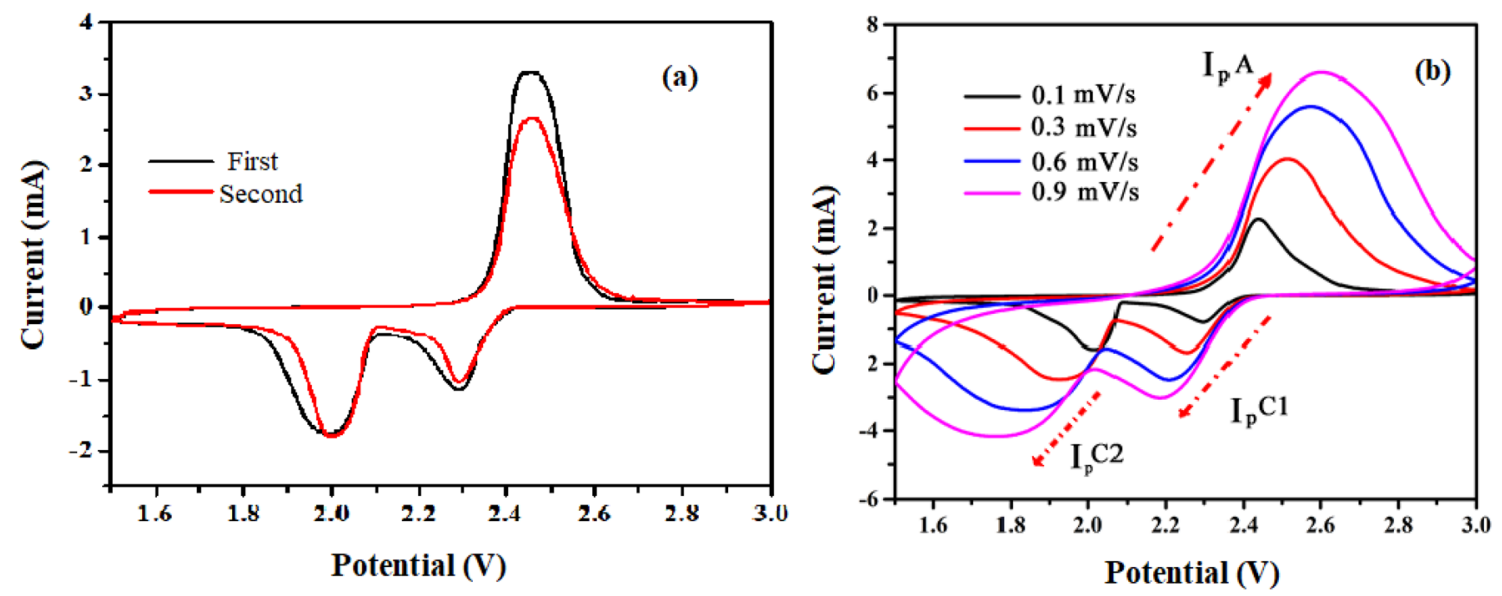

Fig. $4 \mathrm{CV}$ of S-DHCS/CNTs cathode at different a cycle numbers, $\mathbf{b}$ scan rates

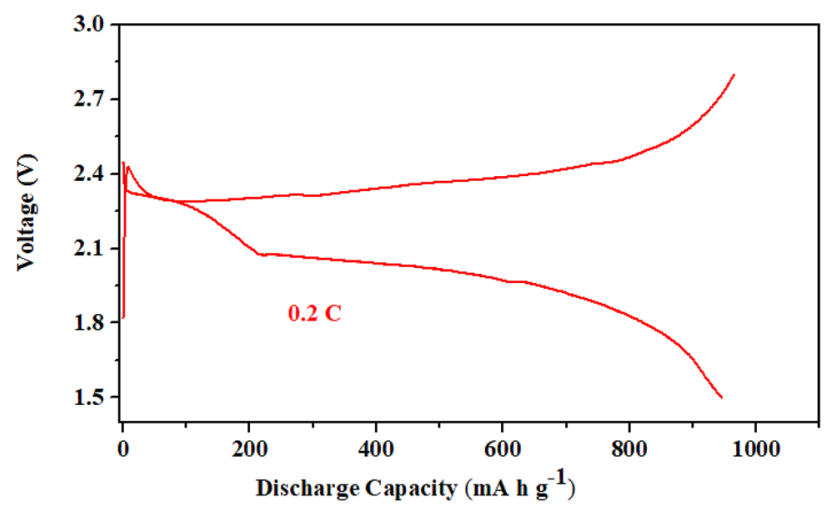

Fig. 5 Charge/discharge profile of S-DHCS/CNTs for LSBs obtained at $0.2 \mathrm{C}$

$\mathrm{CV}$ analysis of the S-DHCS/CNTs cathode was investigated to evaluate the lithium-ion diffusion coefficient $(D)$ of the cathode using the Randles-Sevcik equation [29],

$I_{\mathrm{p}}=2.69 \times 10^{5} n^{3 / 2} A D^{1 / 2} v^{1 / 2} C$,

where $I_{\mathrm{p}}$ is the peak current in ampere (A), $n$ is the number of electrons transferred, $A$ is the surface area $\left(\mathrm{cm}^{2}\right), D$ is diffusion coefficient $\left(\mathrm{cm}^{2} / \mathrm{s}\right), v$ is the scan rate $(\mathrm{V} / \mathrm{s})$, and $C$ is the lithium-ion concentration $\left(\mathrm{mol} / \mathrm{cm}^{3}\right)$.

When the scan rate increases, the charge current also increases and actual capacitance is constant for the materials. This implies that when the scan rate increases, it is allowing more current to flow (i.e., flowing of electrical charge carriers) is indicated in Fig. 4b. The calculated $D$ values are $3.16 \times 10^{-7} \mathrm{~cm}^{2} \mathrm{~s}^{-1}, 4.5 \times 10^{-7} \mathrm{~cm}^{2} \mathrm{~s}^{-1}$ and $6.32 \times 10^{-6} \mathrm{~cm}^{2} \mathrm{~s}^{-1}$ at $2.3,2.02$ and $2.46 \mathrm{~V}$, respectively.

The galvanostatic discharge-charge profile was carried out at a current rate of $0.2 \mathrm{C}$ (Fig. 5). The discharge curve consists of two reduction plateaus about 2.03 and $2.34 \mathrm{~V}$, respectively; while the charge curve consists of one oxidation plateau about $2.4 \mathrm{~V}$. This information is similar to the data obtained by $\mathrm{CV}$.

The electrochemical properties of the cathode prepared from S-DHCS/CNTs are shown in Fig. 6. To study the cyclic and rate performances of the S-DHCS/CNTs cathode material, the $\mathrm{Li}-\mathrm{S}$ batteries using $\mathrm{S}-\mathrm{DHCS} / \mathrm{CNTs}$ cathode material were tested. As shown in Fig. 6a, the initial capacity of S-DHCS/CNTs at $0.2 \mathrm{C}$ is as high as $1709 \mathrm{mAh} \mathrm{g}^{-1}$, and maintain a reversible capacity of about $730 \mathrm{mAh} \mathrm{g}^{-1}$ after 48 cycles with high coulombic efficiency of about $100 \%$. This proposing that the reversibility of $\mathrm{Li}^{+}$insertion and extraction is high [30]. The sulfur loading of S-DHCS/CNTs cathode is $3.3 \mathrm{mg} \mathrm{cm}^{-2}$ with a high areal capacity of 5.6 $\mathrm{mAh} \mathrm{mg} \mathrm{cm}^{-2}$, indicating that the high utilization of the active material. Besides, it could also physically suppress the soluble polysulfides and the volume expansions [31, 32].

The rate performance of the S-DHCS/CNTs cathode was evaluated at different current rates (Fig. 6b). The capacities of the S-DHCS/CNTs cathode are 551, 574, 548, and 840 $\mathrm{mAh} \mathrm{g}^{-1}$ at the high current rate of $3,4,5$, and $0.5 \mathrm{C}$, respectively. When the current rate of S-DHCS/CNTs is restored to $3 \mathrm{C}$, the electrode produces a highly stable specific capacity of $573 \mathrm{mAh} \mathrm{g}^{-1}$. Thus, the S-DHCS/CNTs cathode provides good conductivity and material utilization with $3.0 \mathrm{mg} \mathrm{cm}^{-2}$ sulfur loading.

Thus, the CFP fibers provide better conductivity and better active material utilization was achieved at high cycling rates.

The comparison of the cycling performance of the S-DHCS/CNTs and cathodes at $0.5 \mathrm{C}$ with $3.2 \mathrm{mg} \mathrm{cm}^{-2}(1$ $\mathrm{C}=1675 \mathrm{~mA} \mathrm{~h} \mathrm{~g}^{-1}$ ) was studied (see Fig. 6c). The S-DHCS/ CNTs electrode delivers an initial capacity of $1224 \mathrm{~mA} \mathrm{~h} \mathrm{~g}^{-1}$ with the areal capacity of $5.4 \mathrm{mAh} \mathrm{mg} \mathrm{cm}{ }^{-2}$ and maintains $615 \mathrm{mAh} \mathrm{g}^{-1}$ after 160 cycles, elucidating that the cathode possesses excellent electrochemical performance 

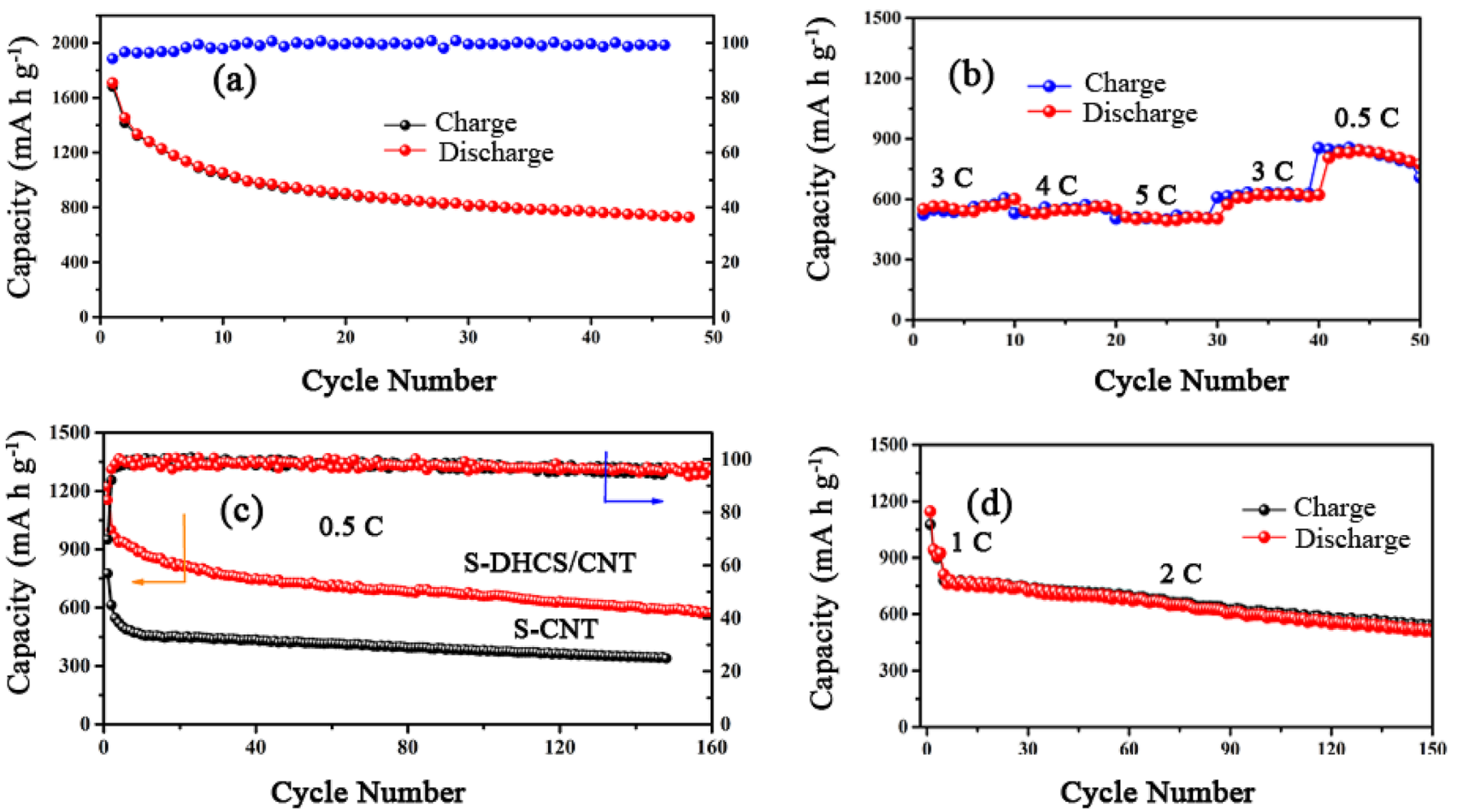

Fig. 6 Electrochemical tests of S-DHCS/CNTs. a Cyclic performance, b rate performance at different rates, $\mathbf{c}$ long cyclic performances of S-DHCS/CNTs and S-CNTs, and $\mathbf{d}$ cyclic performances at $2 \mathrm{C}$ after activation at $1 \mathrm{C}$

in respect of the reversible capacity and cycling stability (stable and higher coulombic efficiency). However, the ordinary S-CNTs electrode shows lower cyclability and lower coulombic efficiency under similar test conditions. Besides, the S-CNTs cathode exhibits rapid capacity fading and the reversible capacity decreases to $339 \mathrm{~mA} \mathrm{~g}^{-1}$ after 148 cycles. The superior capacity of S-DHCS/CNTs is due to the double-hollow structure of the carbon spheres interconnected with CNTs, which enhances the encapsulation of the active material. The novel structure could also suppress the volume expansion and outward diffusion of soluble polysulfides resulting a high coulombic efficiency. Figure 6d shows that both the capacity and cycle performance obtained by the S-DHCS/CNTs in our study surpass many other related works on S-DHCS/CNTs, delivered a high initial capacity of $1145 \mathrm{mAh} \mathrm{g}^{-1}$ at $1 \mathrm{C}$ and $507 \mathrm{mAh}$ $\mathrm{g}^{-1}$ at $2 \mathrm{C}$ after 150 cycles.

\section{Conclusion}

In summary, a double-hollow carbon sphere integrated with CNTs as a sulfur host for $\mathrm{Li}-\mathrm{S}$ batteries was prepared. RF resin is used as a carbon precursor and the $\mathrm{SiO}_{2}$ spheres are prepared by the in situ method. The unique DHCS/CNTs structure can effectively confine sulfur and exhibit high sulfur content (73 wt\%). The CNTs serves as a conductive agent. Owing to the unique structure, the S-DHCS/CNTs electrode performs an outstanding electrochemical performance. The S-DHCS/CNTs cathode with $3.3 \mathrm{mg} \mathrm{cm}^{-2}$ sulfur loading achieves as high as $1709 \mathrm{~mA} \mathrm{~h} \mathrm{~g}^{-1}$, and maintains a reversible capacity of about 730 after 48 cycles at $0.2 \mathrm{C}$ with high coulombic efficiency of about $100 \%$. Besides, the cathode with $3.2 \mathrm{mg} \mathrm{cm}^{-2}$ sulfur loading provides a high areal capacity of $5.4 \mathrm{~mA} \mathrm{~h} \mathrm{mg} \mathrm{cm}{ }^{-2}$. This attributed that the DHCS/CNTs effectively confine sulfur and physically suppress the soluble polysulfides during cycling. Therefore, our work demonstrates the potential application of the material with high sulfur loading for high-performance $\mathrm{Li}-\mathrm{S}$ batteries.

Acknowledgments This work was financially supported by the National Natural Science Foundation of China (Nos. 21636010 and 21878342).

\section{Compliance with ethical standards}

Conflict of interest The authors declare no conflict of interest.

Open Access This article is licensed under a Creative Commons Attribution 4.0 International License, which permits use, sharing, adaptation, distribution and reproduction in any medium or format, as long as you give appropriate credit to the original author(s) and the source, provide a link to the Creative Commons licence, and indicate if changes were made. The images or other third party material in this article are included in the article's Creative Commons licence, unless indicated 
otherwise in a credit line to the material. If material is not included in the article's Creative Commons licence and your intended use is not permitted by statutory regulation or exceeds the permitted use, you will need to obtain permission directly from the copyright holder. To view a copy of this licence, visit http://creativecommons.org/licenses/by/4.0/.

\section{References}

1. Enescu, D., Chicco, G., Porumb, R., Seritan, G.: Thermal energy storage for grid applications: current status and emerging trends. Energies 13(2), 340 (2020)

2. Xiong, C., Ren, Y.X., Jiang, H.R., Wu, M.C., Zhao, T.S.: Artificial bifunctional protective layer composed of carbon nitride nanosheets for high performance lithium-sulfur batteries. J. Energy Storage 26, 101006 (2019)

3. Chen, C., Jiang, J., He, W., Lei, W., Hao, Q., Zhang, X.: 3D printed high-loading lithium-sulfur battery toward wearable energy storage. Adv. Energy Mater. 30(10), 1909469 (2020)

4. Wang, L., Song, Y.H., Zhang, B.H., Liu, Y.T., Wang, Z.Y., Li, G.R., Liu, S., Gao, X.P.: Spherical metal oxides with high tap density as sulfur host to enhance cathode volumetric capacity for lithium-sulfur battery. ACS Appl. Mater. Interfaces 12(5), 5909-5919 (2020)

5. Xu, X., Wang, S., Wang, H., Xu, B., Hu, C., Jin, Y., Liu, J., Yan, H.: The suppression of lithium dendrite growth in lithium sulfur batteries: a review. J Energy Storage 13, 387-400 (2017)

6. Hua, X., Zhang, T., Offer, G.J., Marinescu, M.: Towards online tracking of the shuttle effect in lithium sulfur batteries using differential thermal voltammetry. J. Energy Storage 21, 765-772 (2019)

7. Xi, Y., Angulakshmi, N., Zhang, B., Tian, X., Tang, Z., Xie, P., Chen, G.Z., Zhou, Y.: A $\mathrm{Co}_{9} \mathrm{~S}_{8}$ microsphere and N-doped carbon nanotube composite host material for lithium-sulfur batteries. J. Alloys Compd. 826, 154201 (2020)

8. Kzlaslan, A., Efe, Ü., Akbulut, H.: Electrochemical evaluation of different graphene/sulfur composite synthesis routes in allsolid-state lithium-sulfur batteries. J. Solid State Electrochem. 24, 2279-2288 (2020)

9. Bao, W., Su, D., Zhang, W., Guo, X., Wang, G.: 3D metal carbide@mesoporous carbon hybrid architecture as a new polysulfide reservoir for lithium-sulfur batteries. Adv. Energy Mater. 26(47), 8746-8756 (2016)

10. Wang, X., Sun, Z., Zhao, Y., Li, J., Zhang, Y., Zhang, Z.: $\mathrm{Na}_{4} \mathrm{Mn}_{9} \mathrm{O}_{18}$ nanowires wrapped by reduced graphene oxide as efficient sulfur host material for lithium/sulfur batteries. J. Solid State Electrochem. 24, 111-119 (2020)

11. Evers, S., Nazar, L.F.: New approaches for high energy density lithium-sulfur battery cathodes. Acc. Chem. Res. 46, 1135-1143 (2013)

12. Zheng, B., Yu, L., Li, N., Xi, J.: Efficiently immobilizing and converting polysulfide by a phosphorus doped carbon microtube textile interlayer for high-performance lithium-sulfur batteries. Electrochim. Acta 345, 136186 (2020)

13. Walle, M.D., Zeng, K., Zhang, M., Chabu, J.M., Li, Y., Liu, Y.-N.: Soft template synthesis of acetylene black/manganese dioxide nanosheets composites as efficient sulfur hosts for lithium-sulfur batteries. J. Mater. Sci. 53(20), 14608-14618 (2018)

14. Zhang, B., Luo, C., Deng, Y., Huang, Z., Zhou, G., Lv, W., He, Y.B., Wan, Y., Kang, F., Yang, Q.H.: Optimized catalytic $\mathrm{WS}_{2}-$ $\mathrm{WO}_{3}$ heterostructure design for accelerated polysulfide conversion in lithium-sulfur batteries. Adv. Energy Mater. 10(15), 2000091 (2020)
15. Walle, M.D., Zeng, K., Zhang, M., Li, Y., Liu, Y.-N.: Flower-like molybdenum disulfide/carbon nanotubes composites for high sulfur utilization and high-performance lithium-sulfur battery cathodes. Appl. Surf. Sci. 473, 540-547 (2019)

16. Boyjoo, Y., Shi, H., Olsson, E., Cai, Q., Wu, Z.S., Liu, J., Lu, G.Q.: Molecular-level design of pyrrhotite electrocatalyst decorated hierarchical porous carbon spheres as nanoreactors for lithium-sulfur batteries. Adv. Energy Mater. 10, 2000651 (2020)

17. Walle, M.D., Zhang, Z., Zhang, M., You, X., Li, Y., Liu, Y.-N.: Hierarchical 3D nitrogen and phosphorous codoped graphene/ carbon nanotubes-sulfur composite with synergistic effect for high performance of lithium-sulfur batteries. J. Mater. Sci. 53(4), 2685-2696 (2017)

18. Jin, F., Xiao, S., Lu, L., Wang, Y.: Efficient activation of highloading sulfur by small CNTs confined inside a large CNT for high-capacity and high-rate lithium-sulfur batteries. Nano Lett. 16(1), 440-447 (2016)

19. Zheng, G., Zhang, Q., Cha, J.J., Yang, Y., Li, W., Seh, Z.W., Cui, Y.: Amphiphilic surface modification of hollow carbon nanofibers for improved cycle life of lithium sulfur batteries. Nano Lett. 13(3), 1265-1270 (2013)

20. Chen, H., Dong, W., Ge, J., Wang, C., Wu, X., Lu, W., Chen, L.: Ultrafine sulfur nanoparticles in conducting polymer shell as cathode materials for high performance lithium-sulfur batteries. Sci. Rep. 3(1), 1910 (2013)

21. Zhang, C.Z., Wu, H.B., Yuan, C.Z., Guo, Z.P., Lou, X.W.: Confining sulfur in double-shelled hollow carbon spheres for lithiumsulfur batteries. Angew. Chem. Int. Ed. 124, 9730-9733 (2012)

22. Li, H., Liu, D., Zhu, X., Qu, D., Xie, Z., Li, J., Tang, H., Zheng, D., Qu, D.: Integrated 3D electrodes based on metal-nitrogendoped graphitic ordered mesoporous carbon and carbon paper for high-loading lithium-sulfur batteries. Nano Energy 73, 104763 (2020)

23. Wu, Z., Yao, S., Guo, R., Li, Y., Zhang, C., Shen, X., Li, T., Qin, S.: Freestanding graphitic carbon nitride-based carbon nanotubes hybrid membrane as electrode for lithium/polysulfides batteries. Int. J. Energy Res. 44(4), 3110-3121 (2020)

24. Chen, F., Yang, J., Bai, T., Long, B., Zhou, X.: Biomass wastederived honeycomb-like nitrogen and oxygen dual-doped porous carbon for high performance lithium-sulfur batteries. Electrochim. Acta 192, 99-109 (2016)

25. Chang, N., Zhou, C., Fu, H., Zhao, Y., Shui, J.: One-pot synthesis of functionalized holey graphene/sulfur composite for lithiumsulfur batteries. Adv. Mater. Interfaces 4(21), 1700783 (2017)

26. Abdul Razzaq, A., Yao, Y., Shah, R., Qi, P., Miao, L., Chen, M., Zhao, X., Peng, Y., Deng, Z.: High-performance lithium sulfur batteries enabled by a synergy between sulfur and carbon nanotubes. Energy Storage Mater. 16, 194-202 (2019)

27. Mačák, M., Vyroubal, P., Kazda, T., Jaššo, K.: Numerical investigation of lithium-sulfur batteries by cyclic voltammetry. J. Energy Storage 27, 101158 (2020)

28. Su, W., Feng, W., Wang, S., Chen, L., Song, C.: Electrocatalysis of polysulfide conversion via sulfur-cobalt $\mathrm{CoS}_{2}$ on a carbon nanotube surface as a cathode for high-performance lithium-sulfur batteries. J. Solid State Electrochem. 23(5), 2097-2105 (2019)

29. Kim, H., Lee, J., Ahn, H., Kim, O., Park, M.J.: Synthesis of threedimensionally interconnected sulfur-rich polymers for cathode materials of high-rate lithium-sulfur batteries. Nat. Commun. 6, 7278 (2015)

30. Peng, Y., Peng, Z., Qiu, Y., Yan, K., Wang, G.: Improved performance of lithium-sulfur batteries at elevated temperature by porous aluminum. J. Energy Storage 27, 101104 (2020)

31. Shi, H., Lv, W., Zhang, C., Wang, D.W., Ling, G., He, Y., Kang, F., Yang, Q.H.: Functional carbons remedy the shuttling of polysulfides in lithium-sulfur batteries: confining, trapping, blocking, and breaking up. Adv. Energy Mater. 28(38), 1800508 (2018)

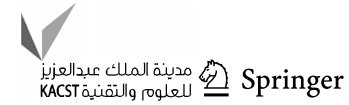


32. Yang, R., Gong, S.M., Zhao, S.Q., Zhao, C.R., Zhang, L.: S@ CNT-graphene-TiN multi-dimensional composites with high sulfur content as the high-performance lithium-sulfur battery cathode materials. J. Solid State Electrochem. 24(9), 1397-1404 (2020)
Publisher's Note Springer Nature remains neutral with regard to jurisdictional claims in published maps and institutional affiliations. 